the most frequent vascular manifestation in BD. In this study, we looked at the pattern and outcome of venous events in BD.

Methods: Seventy five patients, who fulfilled the criteria of the International Study Group for diagnosis of BD, were recruited. We studied the characteristics of patients with thrombotic venous events. Clinical data parameters were recorded, including age at onset, the vascular and extra-vascular manifestations of the disease.

Results: Twenty six from 75 patients had vascular event. Twenty three of these patients had a venous event. The mean age of the patients at the first venous event was 32 years. There were 22 males and 4 females. The first venous event occurred before BD diagnosis in one case and in the same time of onset of the disease in two cases. In the other cases, venous event occurred in patients followed for $\mathrm{BD}$ and the mean disease duration was 5, 82 years. The mean number of recurrence of venous events was 1,46 . Deep vein thrombosis was the most frequent single vascular event $(76,92 \%)$. The most frequent localizations were in legs (23 cases). Four patients had cerebral vein thrombosis. A pulmonary venousinvolvement, a Budd-Chiari syndrome, an inferior and superior vena cava syndrome and arms thrombosis were found in only one case each one. An arterial event was associated in 2 cases. An association with ocular manifestations was observed in $26,9 \%$ patients and neurologic manifestations in $11,53 \%$.

Conclusions: Although there is no agreement on the frequency rate of the vascular lesions in the litterature, most the vascular lesions in the litterature, most of the reported series indicate that the venous lesions are by far, more common than the arterial lesions. Kabbaj et al. reported $85 \%$ venous, $10 \%$ arterial and $5 \%$ mixed arterial and venous involvment which is similar to the reported frequency in our patients.

In conclusion, the frequency of vascular complications of BD in our patients is similar to those reported around the world.

References:

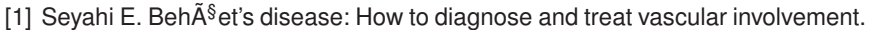

[2] Best Pract Res Clin Rheumatol. 2016 Apr;30(2):279-295.

[3] Seyahi E. BehÃß et's disease: How to diagnose and treat vascular involvement.Best Pract Res Clin Rheumatol. 2016 Apr;30(2):279-295.

[4] Balta S, Balta I, Ozturk C, Celik T, lyisoy A. Beh $\tilde{A}^{\S}$ et's disease and risk of vascular events. Curr Opin Cardiol. 2016 Jul;31(4):451-7.

[5] Seyahi E, Cakmak OS, Tutar B, Arslan C, Dikici AS, Sut N, Kantarci F, Tuzun $H$, Melikoglu M, Yazici $H$. Clinical and Ultrasonographic Evaluation of Lower-extremity Vein Thrombosis in Behcet Syndrome: An Observational Study. Medicine (Baltimore). 2015 Nov;94(44):e1899.

Disclosure of Interest: None declared

DOI: 10.1136/annrheumdis-2017-eular.6850

\section{AB0575 RETROSPECTIVE SURVEY OF CONCOMITANT AUTOIMMUNE DISEASES AND AUTOANTIBODIES IN A COHORT OF PATIENTS WITH ANCA-ASSOCIATED VASCULITIS (AAV)}

M. Casal Moura $^{1,2,3}$, S. Prieto-González ${ }^{3}$, G. Espígol-Frigolé ${ }^{3}$, G. Murgia $^{3,4}$, M. Alba ${ }^{3}$, J. Hernández-Rodríguez ${ }^{3}, \mathrm{M}$. Cid ${ }^{3} .{ }^{1}$ Department of Internal Medicine, São João Hospital Center; ${ }^{2}$ Department of Medicine, Porto Medical School, Porto, Portugal; ${ }^{3}$ Department of Autoimmune Diseases, Hospital Clínic. University of Barcelona. IDIBAPS, Vasculitis Research Unit, Barcelona, Spain; ${ }^{4}$ University Clinic for Visceral Surgery and Medicine, Bauchzentrum Bern, Bern, Switzerland

Background: Anti-neutrophil cytoplasmic antibodies (ANCA) associated vasculitis (AAV) - granulomatosis with polyangiitis (GPA), microscopic polyangiitis (MPA) and eosinophilic granulomatosis with polyangiitis (EGPA) have heterogenous clinic and biologic phenotypes. Sporadic reports indicate that some AAV patients may have other autoimmune diseases but the frequency of the association is unknown.

Objectives: Our purpose was to assess the frequency of other autoimmune diseases or autoantibodies in a well-defined cohort of AAV patients.

Methods: Retrospective survey of a cohort of patients regularly controlled at outpatient facility of an Autoimmune Disease Department in a 5-year period (2011-2016). Clinical and immunologic data were retrieved from electronic records. All patients were diagnosed and treated by the authors.

Results: We included 110 AAV patients - 36 (32.7\%) GPA, 45 (40.9\%) MPA and $29(26.4 \%)$ EGPA. Regarding to ANCA specificity - 23 (20.9\%) against proteinase 3 (PR3)-ANCA, 68 (61.8\%) against myeloperoxidase (MPO)-ANCA and 19 (17.3\%) were ANCA negative. Organ-specific auto-immune diseases was actively searched in $73(81.1 \%)$ patients and was present in $43(58.9 \%)$ patients. More prevalent autoimmune diseases diagnosed were: hypothyroidism (15,34.9\%), pernicious anemia $(12,27.9 \%)$, Sjögren syndrome $(8,18.6 \%)$, hyperthyroidism $(4,9.3 \%)$ and primary biliar cholangitis $(3,7.0 \%)$. Autoantibodies more frequently positive were: ANAs 29 (67.4\%), ASMA 27 (62.8\%), anti-parietal cells 20 (46.5\%), anti-thyreoglobulin 6 (14.0\%), anti-peroxidase $12(27.9 \%)$, anti-SSa 5 $(11.6 \%)$, anti-SSb $3(7.0 \%)$ and RF $14(32.6 \%)$. Prevalence of organ-specific autoimmune disease was higher in MPA $(67.4 \mathrm{vs} .32 .6 \%, \mathrm{p}=0.001)$ and ANCA-MPO carriers $(81.4 \mathrm{vs} .18 .6 \%, \mathrm{p}<0.001)$. Pernicious anemia was more prevalent in MPA $(41.4 \mathrm{vs} .0 \%, \mathrm{p}=0.027)$ and in ANCA-MPO (34.3vs.0\%,p=0.054) and myositis was more prevalent in ANCA-PR3 (12.5vs.0\%,p=0.037). ANAs positivity was more prevalent in ANCA-MPO carriers (74.3\%vs.36.5\%,p=0.048). Predictive factors for development of organ-specific autoimmune disease identified were: female gender (OR 2.710,95\% Cl 1.034-7.099, $\mathrm{p}=0.043$ ), MPA syndrome (OR 3.578, $95 \% \mathrm{Cl} 1.334-9.523, \mathrm{p}=0.011$ ) and MPO carriers (OR 3.346, 95\% Cl 1.1669.601, $\mathrm{p}=0.025)$.

Conclusions: A substantial percentage of AAV patients, particularly MPA and anti MPO carriers, have associated autoimmune diseases and autoantibodies. The limitations of our study (retrospective assessment and lack of comparator) do not allow accurate estimation of prevalence. The severity of AAV and difficulties in management, may lead to overlooking of associated autoimmune diseases which appear to be frequent. Associated autoimmune disease may contribute to additional burden in AAV patients. (Supported by SAF 2014 57708-R).

Disclosure of Interest: None declared

DOI: 10.1136/annrheumdis-2017-eular.1537

\section{AB0576 ANTINEUTROPHILIC CYTOPLASMIC ANTIBODY-ASSOCIATED VASCULITIS: IS HYPOCOMPLEMENTEMIA A PREDICTOR OF POOR PROGNOSIS?}

M. Estévez, A. Argibay, L. Rodriguez, R. Lorenzo, M. Freire,

C. Vázquez-Triñanes, I. Villaverde, B. Gimena, J. Fernández-Martín, A. Rivera. Systemic Autoimmune diseases and Thrombosis Unit, University Hospital Complex of Vigo, Vigo, Spain

Background: Antineutrophil cytoplasmic antibodies (ANCA) associated vasculitis (AAV) is a necrotizing vasculitis that predominantly affects small vessels and is associated with ANCA specific for myeloperoxidase (MPO-ANCA) or proteinase 3 (PR3-ANCA). The major clinicopathologic variants of AAV include microscopic polyangiitis (MPA), granulomatosis with polyangiitis (GPA) and eosinophilic granulomatosis with polyangiitis (EGPA). Recent studies have demonstrated the crucial role of complement activation in the pathogenesis of AAV. However, the clinical characteristics of AAV with hypocomplementemia $(\mathrm{HC})$ still remain unclear. Objectives: The aim of our study was to anlyze the demographic, laboratory, treatment and clinical characteristics of AAV using medical records. To compare the AAV patients with and without $\mathrm{HC}$.

Methods: Retrospective study of patients with AAV diagnosed and followed in a specific Systemic Autoimmune Diseases and Thrombosis Unit. We defined HC as the state in which at least one of the following was lower than the lower limit of the normal range: Complement 3 (C3), Complement 4 (C4). Demographic, clinical, treatment and evolution data were recorded and analyzed using SPSS 22.0.

Results: Thirty-six patients with AAV were included (94,4\% MPO-ANCA, 5,6\% PR3-ANCA). 8 patients were diagnosed of GPA, 21 of MPA and 3 of EGPA. $61,1 \%$ of the patients were males and the mean age at the onset of the disease was $63,11 \pm 14,38$ years [30-85]. $75 \%$ of patients had any vascular risk factors. Renal involvement occurred in 32 patients $(88,9 \%)$ : hematuria in $96,9 \%$, proteinuria in $90,6 \%$ (>1 gr $68,7 \%$ ) and glomerular filtration decreased in $81,25 \%$. Biopsy was performed in 22 patients and a focal and segmental necrotizing glomerulonephritis with extracapillary proliferation was the finding more common. Pulmonary disease $(61,1 \%)$ included interstitial disease $40,9 \%$, alveolar hemorrhage $22,7 \%$ and nodules $18,2 \%$. Other clinical manifestations were constitutional syndrome $36,1 \%$ (the main symptom was asthenia); ear, nose, and throat manifestations $33.3 \%$; neurologic involvement $27.8 \%$ (the main finding was polyneuropathy); articular manifestations $33.3 \%$ and skin lesions $16,7 \%$. All the patients received prednisone in combination with cyclophosphamide $(69.4 \%)$ or rituximab (13.9\%). Azathioprine was used as glucocorticoid-sparing agent (41.7\%). $83.3 \%$ of the patients needed hospital admission and 6 died $(16.7 \%)$. Eleven patients $(30,6 \%)$ had $\mathrm{HC}$ at their diagnosis of AAV. Compared to the AAV patients without $\mathrm{HC}(n=25)$, we found no significant differences in the outcome or severity of the manifestations (evaluated by Five factor score). The small sample size could in part explain these results. Conclusions: Our patients with $\mathrm{HC}$ at diagnosis of AAV did not have different characteristics than those without HC. More studies are needed to determinate if $\mathrm{HC}$ is a predictor of poor prognosis in AAV patients.

Disclosure of Interest: None declared

DOI: 10.1136/annrheumdis-2017-eular.5924

\section{AB0577 SYSTEMIC AUTOIMMUNE VASCULITIS IN RHEUMATOID ARTHRITIS - A POSTMORTEM CLINICOPATHOLOGIC STUDY OF 161 PATIENTS}

M. Bély ${ }^{1}$, Á. Apáthy ${ }^{2} .{ }^{1}$ Department of Pathology, Policlinic of the Order of the Brothers of Saint John of God; ${ }^{2}$ Department of Rheumatology, St. Margaret Clinic Budapest, Budapest, Hungary

Background: Systemic vasculitis of autoimmune origin (A-SV) may be regarded as a basic histological manifestation of rheumatoid arthritis (RA) $[1,2]$.

Objectives: The aim of this study was to characterize the severity of systemic autoimmune vasculitis at death in RA patients.

Methods: A randomized autopsy population of 161 in-patients with RA was studied.

A-SV complicated RA in 32 (19.88\%) patients [3].

RA was confirmed clinically according to the criteria of the ACR [4].

The severity of vasculitis was evaluated by semi-quantitative, visual estimation on a 0 to 3 plus scale in12 organs (heart, lung, liver, spleen, kidney, pancreas, gastrointestinal tract, adrenal gland, skeletal muscle, peripheral nerve, skin and brain) at death) [1]. ("0": no vasculitis, "1": sporadically (scattered) located 